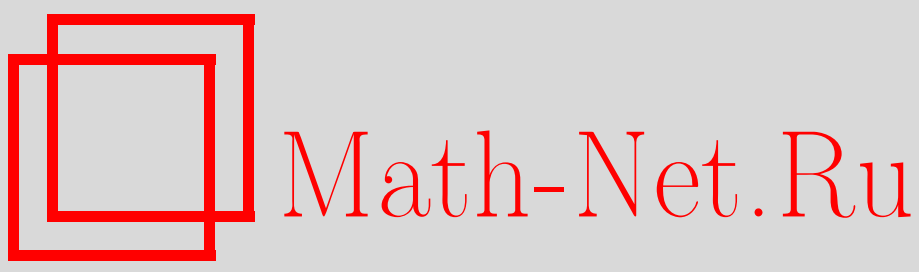

Д. А. Хрычёв, Оптимальное программное управление: существование и аппроксимация, Матем. сб., 2001, том 192, номер 5, 125-144

DOI: https://doi.org/10.4213/sm567

Использование Общероссийского математического портала Math-Net.Ru подразумевает, что вы прочитали и согласны с пользовательским соглашением

http://www . mathnet.ru/rus/agreement

Параметры загрузки:

IP: 54.198 .187 .58

26 апреля 2023 г., 10:08:40 
УДК 517.977

\author{
Д.А. Хрычёв
}

\title{
Оптимальное программное управление: существование и аппроксимация
}

\begin{abstract}
Доказано существование оптимального программного управления для задачи Майера со случайньми параметрами при достаточно общих условиях на динамическую систему. Рассмотрен способ получения субоптимальных управлений, основанньй на дискретизации распределения случайных параметров. Показана возможность распространения указанных результатов на задачи с распределенными параметрами.

Библиограф̆ия: 11 названий.
\end{abstract}

\section{Введение}

Управление стохастической системой, не используюшее информации о ее состоянии и зависящее, следовательно, только от времени, называют программным. Различным вопросам, связанным с программным управлением, посвящена довольно обширная литература; в то же время, этот раздел теории оптимального управления разработан гораздо менее подробно, чем, например, теория управления детерминированной системой или теория адаптивного управления стохастической системой.

В настоящей работе рассмотрены два вопроса: о существовании оптимального программного управления и о приближенном его отыскании. Что касается первого, то здесь автор стремился получить аналоги соответствующих результатов для детерминированных систем [1], [2], по возможности не усиливая налагаемых на задачу ограничений. Следует отметить, что в отношении необходимых условий оптимальности подобная работа уже была проделана в [3].

В основном сформулированное намерение удалось выполнить: в приводимых ниже теоремах сушествования 1.1 и 1.2 имеется лишь одно сушественное ограничение по сравнению с детерминированньм случаем - требование разделения переменной $u$ (управление) и переменных $x$ и $\omega$ (фазовая переменная и случайный параметр) в правых частях уравнений состояния системы (условие д) в теореме 1.1).

Второй вопрос - о субоптимальных управлениях - решается путем перехода от исходной стохастической задачи к детерминированным, получающимся в результате дискретизации распределения случайных параметров (т.е. замены указанного распределения близкими ему атомарными мерами). Оказалось, что эффективность такой аппроксимации существенно зависит от фазовых ограничений. Например, в случае их отсутствия оптимальные (или субоптимальные) управления для приближенных задач образуют минимизирующую последовательность для функционала качества исходной задачи, причем даже при более слабых условиях, чем в теоремах сушествования (в частности, в отсутствие упоминавшегося условия разделения переменных). В то же время, при наличии фазового ограничения вида

$$
x(\cdot) \in X \text { п.н., }
$$

$$
\text { (C) Д. А. Хрычёв } 2001
$$


где $X$ - замкнутое подмножество пространства $C\left(0,1 ; \mathbb{R}^{n}\right)$ траекторий динамической системы, можно гарантировать лишш сходимость минимальных значений функционалов качества приближенных задач к минимуму функционала качества исходной. Рассмотрены также другие случаи фазовых ограничений.

В $\S 2$ показана возможность перенесения описанных результатов на системы с распределенными параметрами. Все рассмотрение проведено на примере нелинейного уравнения гиперболического типа со случайньми правой частью и начальными условиями, управляемого аддитивно входящей в правую часть функцией.

Несколько слов о методах, с помощью которых получены результаты работы.

Методы, использованные при доказательстве теорем существования, частью аналогичны применявшимся в уже упомянутых работах [1], [2] (в разделе, касающемся задач с распределенньпи параметрами, - в работе [4]), частью же примыкают к методам построения статистических решений уравнений с частньми производными, разработанньми М.И. Вишиком с соавторами [5].

Автор не встречал описаний предложенного им в настоящей работе способа аппроксимации оптимального управления. При этом метод дискретизации распределения, лежащий в его основе, неоднократно применялся при решении других задач; в частности, в [6] он был использован при доказательстве разрешимости стохастического гиперболического уравнения, аналогичного приведенному в $\S 2$.

\section{§ 1. Системы, описываемые обыкновенными дифференциальными уравнениями}

Пусть $U$ - метризуемый компакт, $\Omega$ - полное сепарабельное метрическое пространство с метрикой $\rho_{\Omega}$ и $\varphi(t, x, u, \omega)$ - функция со значениями в $\mathbb{R}^{n}$, заданная на $(0,1) \times \mathbb{R}^{n} \times U \times \Omega$.

Рассмотрим задачу

$$
\begin{array}{rlrl}
\dot{x}(t) & =\varphi(t, x(t), u(t), \omega) & & \text { при п.в. } t \in(0,1), \\
x(0) & =x_{0}, & & \\
u(t) & \in U_{t} & \text { при п.в. } t \in(0,1),
\end{array}
$$

где $U_{t}, t \in(0,1)$, - заданные подмножества $U$. Параметр $\omega$ и начальное значение $x_{0}$ предполагаются случайньми, а $u(t)$ играет роль управляющей функции. Таким образом, решение $x(t)$ задачи $(1.1),(1.2)$ (если оно существует) зависит от управления $u=u(\cdot)$ и случайного вектора $\lambda=\left(x_{0}, \omega\right)$ :

$$
x(t)=x(t, u, \lambda) .
$$

Введем функционал качества в форме Майера:

$$
\mathscr{J}(u)=\mathrm{M} g(x(1)) \rightarrow \inf ,
$$

где $g(x)$ - непрерьвная функция $\mathbb{R}^{n} \rightarrow \mathbb{R}_{+}=[0,+\infty), \mathrm{M}$ - оператор математического ожидания.

Задача (1.1)-(1.4) дополняется фазовым ограничением одного из следующих двух видов:

$$
x(\cdot) \in X \text { п.н., }
$$


где $X$ - некоторое подмножество пространства $\mathscr{C}=C\left(0,1 ; \mathbb{R}^{n}\right)^{1}$, либо

$$
\mathfrak{M}_{l}(\cdot) \in X_{l}, \quad l=1,2, \ldots, L,
$$

где $\mathfrak{M}_{l}(t)=\mathrm{M}\left[\bigotimes^{l} x(t)\right], t \in[0,1],-l$-й момент решения $x(t), X_{l}$ - заданное подмножество пространства $\mathscr{C}_{l}=C\left(0,1 ; \bigotimes^{l} \mathbb{R}^{n}\right), l=1,2, \ldots, L$ (пространство $\mathscr{C}_{1}$, очевидно, совпадает с $\mathscr{C}$ ).

Сформулируем условия, обеспечивающие разрешимость задачи (1.1), (1.2). При этом будем использовать запись $K \Subset Y$ для обозначения того факта, что $K$ является компактным подмножеством топологического пространства $Y$.

а) Функция $\varphi(t, x, u, \omega)$ является каратеодориевской по аргументам $t \in(0,1)$ и $(x, u, \omega) \in \mathbb{R}^{n} \times U \times \Omega$ (т.е. при п.в. $t \in(0,1) \varphi(t, x, u, \omega)$ непрерывна по совокупности переменных $x, u, \omega$ и при всех $(x, u, \omega) \in \mathbb{R}^{n} \times U \times \Omega$ измерима по $\left.t\right)$. Для произвольного $R>0$ и каждого $K \Subset \Omega$ сушествует неотрицательная функция $f_{R, K}(t) \in L_{1}(0,1)$ такая, что при п.в. $t \in(0,1)|\varphi(t, x, u, \omega)| \leqslant f_{R, K}(t) \forall u \in U$, $x \in B_{R}=\{|x| \leqslant R\}$ и $\omega \in K$.

б) Для произвольных $R>0$ и $K \Subset \Omega$ сушествует функция $d_{R, K}(t) \in L_{1}(0,1)$ такая, что при п.в. $t \in(0,1)\left|\varphi(t, x, u, \omega)-\varphi\left(t, x^{\prime}, u, \omega\right)\right| \leqslant d_{R, K}(t)\left|x-x^{\prime}\right| \forall u \in U$, $x, x^{\prime} \in B_{R}$ и $\omega \in K$.

в) Сушествуют измеримые по Борелю неотрицательные функции $V(x, \omega)$ и $c(\omega)$, ограниченные на компактных подмножествах пространств $\mathbb{R}^{n} \times \Omega$ и $\Omega$ соответственно, такие, что $V(\cdot, \omega) \in C^{1}\left(\mathbb{R}^{n}\right)$ при каждом фиксированном $\omega \in \Omega$, $V(x, \omega) \rightarrow \infty$ при $|x| \rightarrow \infty$ равномерно по $\omega$, пробегающему компактные подмножества $\Omega$, и при п.в. $t \in(0,1) \quad \nabla_{x} V(x, \omega) \cdot \varphi(t, x, u, \omega) \leqslant c(\omega) V(x, \omega)$ $\forall u \in U, x \in \mathbb{R}^{n}$ и $\omega \in \Omega$.

Выполнение условий а)-в) гарантирует существование на отрезке $[0,1]$ и единственность решения $x(t, u, \lambda)$ задачи $(1.1),(1.2)$ при любой измеримой управляющей функции $u=u(t)$ со значениями в $U$ и любом $\lambda=\left(x_{0}, \omega\right) \in \mathbb{R}^{n} \times \Omega$. В дальнейшем множество измеримых управлений $u:(0,1) \rightarrow U$ будем обозначать через $\mathcal{U}$, a условия а)-в) будем считать выполненньми на протяжении всего параграфа.

Следующая лемма доказывается применением стандартных методов оценивания.

ЛЕмма 1.1. Для произвольного $R>0$ и кажсдого $K \Subset \Omega$ найдутся константы $M$ и $C>0$ и монотонно возрастающие на $\mathbb{R}_{+}$функиии $\xi(\varepsilon)$ и $\zeta(\varepsilon)$, стремящиеся $\kappa$ нулю при $\varepsilon \rightarrow 0+$, такие, что при любом управлении $и \in \mathscr{U}$ и любъх $t, t^{\prime} \in[0,1], \lambda=\left(x_{0}, \omega\right), \lambda^{\prime}=\left(x_{0}^{\prime}, \omega^{\prime}\right) \in B_{R} \times K$ выполняются неравенcmsa

$$
\begin{gathered}
|x(t, u, \lambda)| \leqslant M \\
\left|x(t, u, \lambda)-x\left(t^{\prime}, u, \lambda^{\prime}\right)\right| \leqslant C\left|x_{0}-x_{0}^{\prime}\right|+\xi\left(\rho_{\Omega}\left(\omega, \omega^{\prime}\right)\right)+\zeta\left(\left|t-t^{\prime}\right|\right) .
\end{gathered}
$$

СлЕДСТВИЕ 1.1. Для произвольного управления $u \in \mathscr{U}$ отображсение $\lambda \rightarrow$ $x(\cdot, u, \lambda)$ действует непрерьвно из $\mathbb{R}^{n} \times \Omega$ в $\mathscr{C}$.

Условимся обозначать символом $\mathscr{B}(Y)$ борелевскую $\sigma$-алгебру топологического пространства $Y$. Пусть $\Lambda=\mathbb{R}^{n} \times \Omega$ - пространство случайных параметров задачи $(1.1),(1.2), \mu$ - распределение на $\mathscr{B}(\Lambda)$ случайного вектора $\lambda=\left(x_{0}, \omega\right)$. Для

\footnotetext{
${ }^{1}$ Здесь и ниже $C(0,1 ; V)$ - пространство непрерывных функций, заданных на отрезке [0, 1$]$, со значениями в метрическом пространстве $V$.
} 
произвольного управления $u \in \mathscr{U}$ обозначим через $\Pi_{u}$ совместное распределение на $\mathscr{B}(\Lambda \times \mathscr{C})$ случайного вектора $\lambda$ и решения $x(\cdot, u, \lambda)$ :

$$
\Pi_{u}(B)=\mu\{\lambda:(\lambda, x(\cdot, u, \lambda)) \in B\}, \quad B \in \mathscr{B}(\Lambda \times \mathscr{C}) .
$$

Лемма 1.2. Семейство мер $\left\{\Pi_{u}: u \in \mathscr{U}\right\}$ слабо компактно.

ДокАЗАТЕЛьство. Пусть $\varepsilon>0$ произвольно, и пусть $R_{\varepsilon}>0$ и $K_{\varepsilon} \Subset \Omega$ таковы, что $\mu\left(B_{R_{\varepsilon}} \times K_{\varepsilon}\right)>1-\varepsilon$. Для каждого $u \in \mathscr{U}$ определим множество

$$
Q_{\varepsilon, u}=\left\{(\lambda, x(\cdot, u, \lambda)): \lambda \in B_{R_{\varepsilon}} \times K_{\varepsilon}\right\} \in \mathscr{B}(\Lambda \times \mathscr{C})
$$

и положим $Q_{\varepsilon}=\bigcup_{u \in \mathscr{U}} Q_{\varepsilon, u}$. Из леммы 1.1 с учетом теоремы Арцела следует, что множество $Q_{\varepsilon}$ подкомпактно, а его замыкание $\bar{Q}_{\varepsilon}$, следовательно, компактно в $\Lambda \times \mathscr{C}$. Далее, для любого управления $u \in \mathscr{U} \Pi_{u}\left(\bar{Q}_{\varepsilon}\right) \geqslant \Pi_{u}\left(Q_{\varepsilon, u}\right)=$ $\mu\left(B_{R_{\varepsilon}} \times K_{\varepsilon}\right)$, и доказательство леммы завершается ссылкой на теорему Прохорова [7; теорема 6.1].

Для произвольного управления $u \in \mathscr{U}$ рассмотрим оператор $\mathscr{A}_{u}$, действующий на вектор $(\lambda, x)=\left(x_{0}, \omega, x\right) \in \Lambda \times \mathscr{C}$ по формуле

$$
\mathscr{A}_{u}(\lambda, x)=\left(\dot{x}(\cdot)-\varphi(\cdot, x(\cdot), u(\cdot), \omega), x(0)-x_{0}\right)
$$

Легко проверяется, что оператор $\mathscr{A}_{u}$ действует непрерывно из пространства $\Lambda \times \mathscr{C}$ в $H^{-1}\left(0,1 ; \mathbb{R}^{n}\right) \times \mathbb{R}^{n}$, где $H^{-1}\left(0,1 ; \mathbb{R}^{n}\right)$ - соболевское пространство [8] функций, заданных на интервале $(0,1)$, со значениями в $\mathbb{R}^{n}$.

Пусть, далее, $\pi: \Lambda \times \mathscr{C} \rightarrow \Lambda-$ проекция, $\pi(\lambda, x)=\lambda$.

ЛЕмма 1.3. Борелевская мера $\Pi$ на $\Lambda \times \mathscr{C}$ совпадает с распределением $\Pi_{u}$, определенным в (1.9), тогда и только тогда, когда

$$
\mathscr{A}_{u}^{*} \Pi=\delta_{0} \quad u \quad \pi^{*} \Pi=\mu,
$$

әде $\delta_{0}-$ единичная мера, сосредоточенная в нуле пространства $H^{-1}\left(0,1 ; \mathbb{R}^{n}\right)$ $\times \mathbb{R}^{n}, \mathscr{A}_{u}^{*} \Pi u \pi^{*} \Pi-$ образы мерь $\Pi$ при отображсениях $\mathscr{A}_{u} u \pi:$

$$
\begin{array}{ll}
\mathscr{A}_{u}^{*} \Pi(B)=\Pi\left(\mathscr{A}_{u}^{-1}(B)\right) & \forall B \in \mathscr{B}\left(H^{-1}\left(0,1 ; \mathbb{R}^{n}\right) \times \mathbb{R}^{n}\right), \\
\pi^{*} \Pi\left(B^{\prime}\right)=\Pi\left(\pi^{-1}\left(B^{\prime}\right)\right) & \forall B^{\prime} \in \mathscr{B}(\Lambda) .
\end{array}
$$

ДоКАЗАТЕЛЬСтво. Если $\Pi=\Pi_{u}$, то (1.11) очевидно. Обратно, пусть для меры П справедливы равенства (1.11). Обозначим через $\Gamma_{u}$ график отображения $\lambda \rightarrow x(\cdot, u, \lambda):$

$$
\Gamma_{u}=\{(\lambda, x(\cdot, u, \lambda)): \lambda \in \Lambda\}=\left\{(\lambda, x) \in \Lambda \times \mathscr{C}: \mathscr{A}_{u}(\lambda, x)=0\right\} \in \mathscr{B}(\Lambda \times \mathscr{C}) .
$$

В силу первого из соотношений (1.11)

$$
\Pi\left(\Gamma_{u}\right)=\Pi\left(\mathscr{A}_{u}^{-1}(0)\right)=\delta_{0}(0)=1
$$

а потому для произвольного множества $B \in \mathscr{B}(\Lambda \times \mathscr{C})$

$$
\begin{aligned}
\Pi(B) & =\Pi\left(B \cap \Gamma_{u}\right)=\Pi\left(\pi^{-1}\left(\pi\left(B \cap \Gamma_{u}\right)\right) \cap \Gamma_{u}\right) \\
& =\Pi\left(\pi^{-1}\left(\pi\left(B \cap \Gamma_{u}\right)\right)\right)=\pi^{*} \Pi\left(\pi\left(B \cap \Gamma_{u}\right)\right) \\
& =\mu\left(\pi\left(B \cap \Gamma_{u}\right)\right)=\mu\{\lambda:(\lambda, x(\cdot, u, \lambda)) \in B\},
\end{aligned}
$$


откуда в силу (1.9) имеем $\Pi=\Pi_{u}$. Отметим, что измеримость по Борелю множества $\pi\left(B \cap \Gamma_{u}\right)$ (а значит, и множества $\left.\pi^{-1}\left(\pi\left(B \cap \Gamma_{u}\right)\right)\right)$ вытекает из непрерывности отображения $\lambda \rightarrow x(\cdot, u, \lambda)$, так как $\pi\left(B \cap \Gamma_{u}\right)=\{\lambda:(\lambda, x(\cdot, u, \lambda)) \in B\}$.

Назовем управление $u \in \mathscr{U}$ допустимым, если:

1) оно удовлетворяет условию (1.3);

2) $\mathscr{J}(u)<\infty$;

$3)$ решение $x(t, u, \lambda)$ удовлетворяет фазовому ограничению (1.5) или (1.6) в случае его наличия.

Множество допустимых управлений обозначим через $\mathscr{U}_{d}$. Будем предполагать выполненным условие

г) множество $\mathscr{U}_{d}$ непусто.

Докажем теорему о сушествовании оптимального управления для задачи с фазовым ограничением (1.5).

ТЕОРЕМА 1.1. Пусть помимо б)-г) выполнены условия:

д) функиия $\varphi$ представляется в виде

$$
\varphi(t, x, u, \omega)=\sum_{i=1}^{m} \beta_{i}(t, x, \omega) \chi_{i}(t, u)
$$

әде $\chi_{i}$ и $\beta_{i}, i=1,2, \ldots, m,-$ каратеодориевские функиии со значениями в $\mathbb{R} u \mathbb{R}^{n}$ соответственно;

е) существуют функиии $h_{i}(t) \in L_{p_{i}}(0,1), 1<p_{i} \leqslant \infty$, такие, что при n.s. $t$

$$
\left|\chi_{i}(t, u)\right| \leqslant h_{i}(t) \quad \forall u \in U, \quad i=1,2, \ldots, m
$$

ж) для произвольных $R>0$ и $K \Subset \Omega$ существуют функции $b_{i, R, K}(t) \in$ $L_{q_{i}}(0,1), q_{i}=p_{i} /\left(p_{i}-1\right)\left(q_{i}=1\right.$, если $\left.p_{i}=\infty\right), i=1,2, \ldots, m$, для которых при п.в. $t$

$$
\left|\beta_{i}(t, x, \omega)\right| \leqslant b_{i, R, K}(t) \quad \forall x \in B_{R} \quad u \quad \omega \in K
$$

3) при п.в. $t \in(0,1)$ множества $U_{t}$ замкнутви в $U$;

и) множество $\left\{(t, u): t \in(0,1), u \in U_{t}\right\}$ измеримо по Борелю;

к) при п.в. $t \in(0,1)$ множества $W_{t}=\left\{\left(\chi_{1}(t, u), \chi_{2}(t, u), \ldots, \chi_{m}(t, u)\right)\right.$ : $\left.u \in U_{t}\right\}$ выпукльи;

л) множсество $X$ замкнуто в $\mathscr{C}$.

Тогда существует по меньшей мере одно оптимальное управление для задачи (1.1)-(1.5).

ЗАмЕчАниЕ. Выполнение условия а) следует из д)-ж).

ДоказАТЕЛЬСтво. Рассмотрим минимизируюшую функционал (1.4) последовательность управлений $u_{k} \in \mathscr{U}_{d}$. Пусть $\Pi_{k}=\Pi_{u_{k}}$ - распределения (1.9). Согласно лемме 1.2 и условию е) сушествует подпоследовательность $\left\{k^{\prime}\right\} \subset\{k\}$ такая, что $\Pi_{k^{\prime}} \rightarrow \Pi$ слабо на $\Lambda \times \mathscr{C}$ и $\chi_{i}\left(t, u_{k^{\prime}}(t)\right) \rightarrow \psi_{i}(t)$ слабо в $L_{p_{i}}(0,1), i=1, \ldots, m$

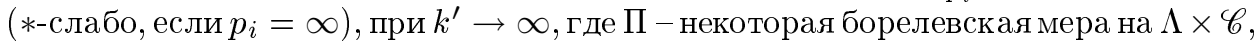


$\psi_{i}$ - некоторая функция из $L_{p_{i}}(0,1)$. При помощи стандартных выкладок, используюших свойства выпуклости и теорему об измеримой выборке [2], доказывается сушествование управления $\widehat{u}(t) \in U_{t}$ такого, что $\psi_{i}(t)=\chi_{i}(t, \widehat{u}(t))$ при п.в. $t$, $i=1,2, \ldots, m$.

Покажем, что распределение П $\widehat{\widehat{u}}$, отвечающее управлению $\widehat{u}$ согласно (1.9), совпадает с предельной мерой П.

Пусть $\left(\lambda_{k}, x_{k}\right) \rightarrow(\lambda, x)$ при $k \rightarrow \infty$ в $\Lambda \times \mathscr{C}$. Из (1.10), условий д)-ж) и полной непрерывности вложения $L_{1}\left(0,1 ; \mathbb{R}^{n}\right) \subset H^{-1}\left(0,1 ; \mathbb{R}^{n}\right)$ заключаем, что

$$
\mathscr{A}_{u_{k^{\prime}}}\left(\lambda_{k^{\prime}}, x_{k^{\prime}}\right) \rightarrow \mathscr{A}_{\widehat{u}}(\lambda, x) \text { в } H^{-1}\left(0,1 ; \mathbb{R}^{n}\right) \times \mathbb{R}^{n}
$$

по выбранной вьше подпоследовательности $\left\{k^{\prime}\right\}$. Отсюда по известному свойству слабой сходимости мер [7; теорема 5.5] следует, что $\mathscr{A}_{u_{k^{\prime}}}^{*} \Pi_{k^{\prime}} \rightarrow \mathscr{A}_{\widehat{u}}^{*} \Pi, k^{\prime} \rightarrow \infty$, слабо на пространстве $H^{-1}\left(0,1 ; \mathbb{R}^{n}\right) \times \mathbb{R}^{n}$. Поскольку $\mathscr{A}_{u_{k}}^{*} \Pi_{k}=\delta_{0}$ в силу леммы 1.3 , то же верно и для предельной меры. Далее, $\pi^{*} \Pi_{k^{\prime}} \rightarrow \pi^{*} \Pi, k^{\prime} \rightarrow \infty$, слабо на $\Lambda$ в силу непрерывности $\pi$ и, значит, $\pi^{*} \Pi=\mu$. Из леммы 1.3 теперь следует, что П совпадает с распределением $\Pi_{\widehat{u}}$.

Покажем, что

$$
\mathscr{J}(\widehat{u}) \leqslant \inf _{u \in \mathscr{U}_{d}} \mathscr{J}(u)
$$

Действительно, из непрерывности и ограниченности снизу на $\mathscr{C}$ функционала $g(x(1))$ и сходимости $\Pi_{k^{\prime}} \rightarrow \Pi_{\widehat{u}}$ получим: ${ }^{2}$

$$
\begin{aligned}
\mathscr{J}(\widehat{u}) & =\int g(x(1)) d \Pi_{\widehat{u}} \leqslant \liminf _{k^{\prime} \rightarrow \infty} \int g(x(1)) d \Pi_{k^{\prime}} \\
& =\liminf _{k^{\prime} \rightarrow \infty} \mathscr{J}\left(u_{k^{\prime}}\right)=\inf _{u \in \mathscr{U}_{d}} \mathscr{J}(u) .
\end{aligned}
$$

Докажем, наконец, что $\widehat{u}$ - допустимое управление. Для этого достаточно установить выполнение фазового ограничения (1.5). Заметим, что в силу определения (1.9) условие (1.5) эквивалентно следующему:

$$
\Pi_{u}(\Lambda \times X)=1
$$

Условие (1.14) выполнено для распределений $\Pi_{k}$, соответствующих $u_{k} \in \mathscr{U}_{d}$, а потому, благодаря замкнутости $X$, и для предельного распределения $\Pi=\Pi_{\widehat{u}}$ :

$$
\Pi_{\widehat{u}}(\Lambda \times X) \geqslant \limsup _{k^{\prime} \rightarrow \infty} \Pi_{k^{\prime}}(\Lambda \times X)=1
$$

Таким образом, $\widehat{u}(t)$ - допустимое, а значит (вследствие $(1.12)$ ), оптимальное управление. Теорема доказана.

Сформулируем теорему сушествования для задачи с фазовым ограничением (1.6).

\footnotetext{
2 Здесь и ниже, если не указана область интегрирования, интеграл берется по всему пространству, на котором задана мера.
} 
ТЕОРЕМА 1.2. Пусть выполнены условия б)-к), а такэсе

$\left.\pi^{\prime}\right)$ множества $X_{l}$ замкнуты в $\mathscr{C}_{l}, l=1, \ldots, L$;

м) существуют полохсительнье константы $D, R_{0}, \rho_{1} u \rho_{2}$ такие, что:

1) $\rho=\rho_{1} \rho_{2}>L$

2) $V(x, \omega) \geqslant D|x|^{\rho_{1}} \quad \forall x:|x| \geqslant R_{0}, \forall \omega \in \Omega$;

3) $\mathrm{M}\left[V\left(x_{0}, \omega\right) e^{c(\omega)}\right]^{\rho_{2}}<\infty$

(функиии $V(x, \omega)$ и с $(\omega)$ введены в условии в)).

Тогда существует по меньшей мере одно оптимальное управление для задачи (1.1)-(1.4), (1.6).

ДоКАЗАТЕЛЬСТво повторяет доказательство теоремы 1.1 вплоть до момента проверки выполнения фазового ограничения, на чем и остановимся.

Из в) и леммы Гронуолла следует, что для произвольных $u \in \mathscr{U}$ и $\lambda=\left(x_{0}, \omega\right)$ решение $x(t)=x(t, u, \lambda)$ задачи (1.1), (1.2) удовлетворяет оценке

$$
V(x(t), \omega) \leqslant V\left(x_{0}, \omega\right) e^{c(\omega) t} \quad \forall t \in[0,1] .
$$

Из (1.15) и условия м) вытекает сушествование моментов $\mathrm{M}\left[\otimes^{l} x(t)\right]$ порядков $l=1,2, \ldots, L$ у всех решений $x(t)$.

Пусть $x_{k}(t)=x\left(t, u_{k}, \lambda\right), \widehat{x}(t)=x(t, \widehat{u}, \lambda)$, где $u_{k}$ и $\widehat{u}-$ такие же управления, как в доказательстве теоремы 1.1. Поскольку $u_{k} \in \mathscr{U}_{d}$, для решений $x_{k}(t)$ ограничение (1.6) выполнено. Покажем, что равномерно на отрезке $[0,1] \mathrm{M}\left[\bigotimes^{l} x_{k}(t)\right] \rightarrow$ $\mathrm{M}\left[\otimes^{l} \widehat{x}(t)\right]$ при $k=k^{\prime} \rightarrow \infty$, откуда в силу замкнутости множеств $X_{l}$ в $\mathscr{C}_{l}$ будет следовать справедливость (1.6) для $\widehat{x}(t)$.

Пусть $x=\left(x^{1}, x^{2}, \ldots, x^{n}\right) \in \mathbb{R}^{n}, \Phi(x)=x^{i_{1}} x^{i_{2}} \cdots x^{i_{l}}$ - некоторая компонента тензора $\bigotimes^{l} x, 1 \leqslant l \leqslant L$. Для произвольного $t \in[0,1]$

$$
\begin{gathered}
\mathrm{M} \Phi\left(x_{k}(t)\right)-\mathrm{M} \Phi(\widehat{x}(t))=\int \Phi(x(t))\left(d \Pi_{k}-d \Pi\right)=\int \Phi_{N}(x(t))\left(d \Pi_{k}-d \Pi\right) \\
+\int\left(\Phi(x(t))-\Phi_{N}(x(t))\right) d \Pi_{k}+\int\left(\Phi_{N}(x(t))-\Phi(x(t))\right) d \Pi,
\end{gathered}
$$

где $\Phi_{N}(x)=\Phi(x)$, если $|\Phi(x)| \leqslant N, \Phi_{N}(x)= \pm N$ при $\Phi(x)>N$ и $\Phi(x)<-N$ соответственно $(N>0)$.

Первый интеграл в правой части (1.16) сходится к нулю при $k=k^{\prime} \rightarrow \infty$ для каждого $N$ в силу слабой сходимости $\Pi_{k^{\prime}} \rightarrow$ П. Второй (и, аналогично, третий) допускает оценку

$$
\begin{aligned}
& \left|\int\left(\Phi(x(t))-\Phi_{N}(x(t))\right) d \Pi_{k}\right| \leqslant \int_{\{|\Phi(x(t))|>N\}}|\Phi(x(t))| d \Pi_{k} \\
& \leqslant \int_{\left\{|x(t)|>N^{1 / l}\right\}}|x(t)|^{l} d \Pi_{k} \leqslant \frac{1}{N^{\rho-l}} \int_{\left\{|x(t)|>N^{1 / l}\right\}}|x(t)|^{\rho} d \Pi_{k} \\
& \leqslant \frac{1}{D^{\rho_{2}} N^{\rho-l}} \int[V(x(t), \omega)]^{\rho_{2}} d \Pi_{k} \leqslant \frac{\mathrm{M}\left[V(\lambda) e^{c(\omega)}\right]^{\rho_{2}}}{D^{\rho_{2}} N^{\rho-l}}
\end{aligned}
$$

для любых $k$ и $N \geqslant R_{0}^{l}$ в силу условия м) и (1.15). Отсюда с учетом м) следует поточечная сходимость $\mathrm{M} \Phi\left(x_{k^{\prime}}(t)\right) \rightarrow \mathrm{M} \Phi(\widehat{x}(t))$. 
Для доказательства равномерности полученной сходимости заметим, что множество функций $\{\mathrm{M} \Phi(x(t))\}$, где $x(t)$ - всевозможные решения задачи $(1.1),(1.2)$, равностепенно непрерьвно. Действительно, пусть $\varepsilon>0$ произвольно, а $R_{\varepsilon}>0$ и $K_{\varepsilon} \Subset \Omega$ таковы, что $\mu\left(B_{R_{\varepsilon}} \times K_{\varepsilon}\right)>1-\varepsilon$. Тогда для произвольного решения $x(t)$

$$
\begin{aligned}
& \left|\mathrm{M} \Phi(x(t))-\mathrm{M} \Phi\left(x\left(t^{\prime}\right)\right)\right| \leqslant \int_{B_{R_{\varepsilon}} \times K_{\varepsilon}}\left|\Phi(x(t, \lambda))-\Phi\left(x\left(t^{\prime}, \lambda\right)\right)\right| d \mu \\
& \quad+\int_{\Lambda \backslash\left(B_{R_{\varepsilon}} \times K_{\varepsilon}\right)}\left(|\Phi(x(t, \lambda))|+\left|\Phi\left(x\left(t^{\prime}, \lambda\right)\right)\right|\right) d \mu \\
& \leqslant l \int_{B_{R_{\varepsilon}} \times K_{\varepsilon}}\left|x(t, \lambda)-x\left(t^{\prime}, \lambda\right)\right| \max _{t}|x(t, \lambda)|^{l-1} d \mu \\
& \quad+\int_{\Lambda \backslash\left(B_{R_{\varepsilon}} \times K_{\varepsilon}\right)}\left(|x(t, \lambda)|^{l}+\left|x\left(t^{\prime}, \lambda\right)\right|^{l}\right) d \mu \\
& \leqslant l M^{l-1} \zeta\left(\left|t-t^{\prime}\right|\right)+2 \varepsilon^{(\rho-l) / \rho}\left(R_{0}^{\rho} \varepsilon+\frac{\mathrm{M}\left[V(\lambda) e^{c(\omega)}\right]^{\rho_{2}}}{D^{\rho_{2}}}\right)^{l / \rho}
\end{aligned}
$$

в силу (1.7), (1.8), условия м) и неравенства Гёльдера. Из полученной оценки следует равностепенная непрерьвность $\{\mathrm{M} \Phi(x(t))\}$, чем и завершается доказательство теоремы.

Перейдем к изложению результатов об аппроксимации. Основная идея предлагаемого метода состоит в замене распределения $\mu$ близкой ему в смысле метрики Прохорова атомарной (т.е. сосредоточенной на конечном множестве точек) мерой $\mu_{j}$, что преврашает рассматриваемую задачу со случайньми параметрами в детерминированную.

Опишем построение атомарных мер $\mu_{j}$.

Пусть $\sigma_{j}$ и $\varepsilon_{j}, j=1,2, \ldots,-$ сходящиеся к нулю последовательности положительных чисел. Для каждого $j$ возьмем компактное в $\Lambda$ подмножество $\mathscr{K}_{j}$ такое, что $\mathscr{K}_{j}$ содержится в носителе $\operatorname{supp} \mu$ меры $\mu$ и $\mu\left(\mathscr{K}_{j}\right)>1-\sigma_{j}$. В $\mathscr{K}_{j}$ выберем конечную $\frac{\varepsilon_{j}}{2}$-сеть $\left\{\lambda_{1}, \lambda_{2}, \ldots, \lambda_{N_{j}}\right\}$.

Пусть, далее, $\varkappa_{j}(t)$ - определенная при $t \geqslant 0$ непрерьвная функция, причем $\varkappa_{j}(t)>0$ при $t<\varepsilon_{j}$ и $\varkappa_{j}(t)=0$ при $t \geqslant \varepsilon_{j}$. Очевидно, функции

$$
\gamma_{i}(\lambda)=\frac{\varkappa_{j}\left(\rho_{\Lambda}\left(\lambda, \lambda_{i}\right)\right)}{\sum_{l=1}^{N_{j}} \varkappa_{j}\left(\rho_{\Lambda}\left(\lambda, \lambda_{l}\right)\right)}, \quad \lambda \in \mathscr{K}_{j}, \quad i=1,2, \ldots, N_{j},
$$

образуют разбиение единицы на $\mathscr{K}_{j}$, причем $\gamma_{i}(\lambda)=0$ при $\rho_{\Lambda}\left(\lambda, \lambda_{i}\right) \geqslant \varepsilon_{j}$ (здесь $\rho_{\Lambda}\left(\lambda, \lambda^{\prime}\right)=\left|x_{0}-x_{0}^{\prime}\right|+\rho_{\Omega}\left(\omega, \omega^{\prime}\right), \lambda=\left(x_{0}, \omega\right), \lambda^{\prime}=\left(x_{0}^{\prime}, \omega^{\prime}\right) \in \Lambda-$ метрика в $\left.\Lambda\right)$.

Обозначим символом $\delta_{\lambda}$ единичную меру, сосредоточенную в точке $\lambda \in \Lambda$, и положим

$$
\mu_{j}=\sum_{i=1}^{N_{j}} \alpha_{i} \delta_{\lambda_{i}}, \quad \text { где } \quad \alpha_{i}=\int_{\mathscr{K}_{j}} \gamma_{i}(\lambda) d \mu .
$$

Рассмотрим задачи (1.1)-(1.5) и (1.1)-(1.4), (1.6) в ситуации, когда их случайные параметры имеют распределение $\mu_{j}$ (меры $\mu_{j}$, вообще говоря, не являются вероятностными, но для краткости в отношении их используется теоретико-вероятностная терминология). Обозначим $x_{i}(t)=x\left(t, u, \lambda_{i}\right)$ и $\varphi_{i}(t, x, u)=$ $\varphi\left(t, x, u, \omega_{i}\right), i=1,2, \ldots, N_{j}$, где $\lambda_{i}=\left(x_{0_{i}}, \omega_{i}\right)$ - элементы выбранной нами 
$\frac{\varepsilon_{j}}{2}$-сети. Пусть $\mathrm{M}_{j}$ - оператор математического ожидания на вероятностном пространстве $\left(\Lambda, \mathscr{B}(\Lambda), \mu_{j}\right)$. Тогда соотношения (1.1)-(1.6) эквивалентны следующим:

$$
\begin{gathered}
\dot{x}_{i}(t)=\varphi_{i}\left(t, x_{i}(t), u(t)\right), \quad i=1,2, \ldots, N_{j} \\
x_{i}(0)=x_{0_{i}}, \quad i=1,2, \ldots, N_{j}, \\
u(t) \in U_{t}, \\
\mathscr{J}_{j}(u)=\mathrm{M}_{j} g(x(1))=\sum_{i=1}^{N_{j}} \alpha_{i} g\left(x_{i}(1)\right) \rightarrow \inf , \\
x_{i}(\cdot) \in X, \quad i=1,2, \ldots, N_{j},
\end{gathered}
$$

и, наконец,

$$
\mathfrak{M}_{l, j}(\cdot) \in X_{l}, \quad l=1,2, \ldots, L,
$$

где $\mathfrak{M}_{l, j}(t)=\mathrm{M}_{j}\left[\bigotimes^{l} x(t)\right]=\sum_{i=1}^{N_{j}} \alpha_{i}\left[\bigotimes^{l} x_{i}(t)\right]$.

Покажем, что полученные детерминированные задачи (1.18)-(1.22) и (1.18)-(1.21), (1.23) в известном смысле аппроксимируют исходные.

ЛЕмма 1.4. Последовательность мер $\mu_{j}$ слабо на пространстве $\Lambda$ сходится $\kappa \mu$.

ДокАЗАТЕЛЬСтво. Достаточно проверить [7], что

$$
\mathrm{M}_{j} \psi=\int \psi(\lambda) d \mu_{j} \underset{j \rightarrow \infty}{\longrightarrow} \mathrm{M} \psi=\int \psi(\lambda) d \mu
$$

для любой ограниченной равномерно непрерьвной на $\Lambda$ функции $\psi(\lambda)$. Согласно (1.17)

$$
\begin{aligned}
\mathrm{M}_{j} \psi-\mathrm{M} \psi & =\int_{\mathscr{K}_{j}} \sum_{i=1}^{N_{j}} \gamma_{i}(\lambda) \psi\left(\lambda_{i}\right) d \mu-\int \psi(\lambda) d \mu \\
& =\int_{\mathscr{K}_{j}} \sum_{i=1}^{N_{j}} \gamma_{i}(\lambda)\left(\psi\left(\lambda_{i}\right)-\psi(\lambda)\right) d \mu-\int_{\Lambda \backslash \mathscr{K}_{j}} \psi(\lambda) d \mu
\end{aligned}
$$

откуда следует оценка

$$
\left|\mathrm{M}_{j} \psi-\mathrm{M} \psi\right| \leqslant m_{\psi}\left(\varepsilon_{j}\right) \mu\left(\mathscr{K}_{j}\right)+\sigma_{j} \max _{\Lambda}|\psi(\lambda)|,
$$

где $m_{\psi}(\varepsilon)=\max _{\rho_{\Lambda}\left(\lambda, \lambda^{\prime}\right) \leqslant \varepsilon}\left|\psi(\lambda)-\psi\left(\lambda^{\prime}\right)\right|-$ модуль непрерывности функции $\psi$, $m_{\psi}(\varepsilon) \rightarrow 0$ при $\varepsilon \rightarrow 0$. Учитывая стремление к нулю $\varepsilon_{j}$ и $\sigma_{j}$, из $(1.24)$ выводим утверждение леммы.

Ниже последовательность $\varepsilon_{j}$ выбирается с таким расчетом, чтобы обеспечить сходимость $\mathrm{M}_{j} g(x(1))$ к $\mathrm{M} g(x(1))$ - функционалу качества (1.4).

Возьмем последовательности чисел $R_{1} \leqslant R_{2} \leqslant \cdots$ и компактов $K_{1} \subset K_{2} \subset$ $\cdots \subset \Omega$ такие, что $\mathscr{K}_{j} \subset B_{R_{j}} \times K_{j}, j=1,2, \ldots$ Пусть $M=M_{j}$ - константа из оценки $(1.7)$, соответствуюшая числу $R_{j}$ и компакту $K_{j}$,

$$
m_{g}^{j}(\varepsilon)=\max \left\{\left|g(x)-g\left(x^{\prime}\right)\right|: x, x^{\prime} \in B_{M_{j}},\left|x-x^{\prime}\right| \leqslant \varepsilon\right\}
$$


- модуль непрерывности функции $g$ на шаре $B_{M_{j}}$. Выберем $\varepsilon_{j}$ таким образом, чтобы

$$
m_{g}^{j}\left(C_{j} \varepsilon_{j}+\xi_{j}\left(\varepsilon_{j}\right)\right) \rightarrow 0 \text { при } j \rightarrow \infty,
$$

где $C=C_{j}$ и $\xi=\xi_{j}$ - соответствующие $R_{j}$ и $K_{j}$ константа и функция из оценки $(1.8)$ (указанный выбор $\varepsilon_{j}$ возможен, поскольку $\xi_{j}(\varepsilon) \rightarrow 0$ и $m_{g}^{j}(\varepsilon) \rightarrow 0$ при $\varepsilon \rightarrow 0)$.

ЛЕмма 1.5. Пусть последовательность $\varepsilon_{j}$ выбрана с учетом требования (1.26). Предположим, далее, что либо функчия $g(x)$ ограничена, либо выполнено условие

н) существуют положстельные константы $r, G$ и $M_{0}$ такие, что

$$
\mathrm{M}\left[V(\lambda) e^{c(\omega)}\right]^{r}<\infty
$$

u $g(x) \leqslant G[V(x, \omega)]^{r} \quad \forall x:|x| \geqslant M_{0}, \forall \omega \in \Omega$. Тогда функциональц $\mathscr{J}_{j}(u)=$ $\mathrm{M}_{j} g(x(1))$ сходятся при $j \rightarrow \infty \kappa \mathscr{J}(u)=\mathrm{M} g(x(1))$ равномерно по всевозможным управлениям $и \in \mathscr{U}$.

ДокАЗАТЕЛЬСТво приведем для случая, когда $g(x)$ неограничена.

Аналогично выкладкам из леммы 1.4

$$
\begin{aligned}
\mathscr{J}_{j}(u)-\mathscr{J}(u)= & \int_{\mathscr{K}_{j}} \sum_{i=1}^{N_{j}} \gamma_{i}(\lambda)\left[g\left(x\left(1, u, \lambda_{i}\right)\right)-g(x(1, u, \lambda))\right] d \mu \\
& -\int_{\Lambda \backslash \mathscr{K}_{j}} g(x(1, u, \lambda)) d \mu,
\end{aligned}
$$

откуда в силу оценок $(1.7),(1.8),(1.15)$ и условия н)

$$
\begin{aligned}
\left|\mathscr{J}_{j}(u)-\mathscr{J}(u)\right| \leqslant & \int_{\mathscr{K}_{j}} \sum_{i} \gamma_{i}(\lambda) m_{g}^{j}\left(\left|x\left(1, u, \lambda_{i}\right)-x(1, u, \lambda)\right|\right) d \mu \\
& +G \int_{\Lambda \backslash \mathscr{K}_{j}}[V(x(1, u, \lambda), \omega)]^{r} d \mu+\max _{|x| \leqslant M_{0}} g(x) \mu\left(\Lambda \backslash \mathscr{K}_{j}\right) \\
\leqslant & m_{g}^{j}\left(C_{j} \varepsilon_{j}+\xi_{j}\left(\varepsilon_{j}\right)\right) \mu\left(\mathscr{K}_{j}\right) \\
& +G \int_{\Lambda \backslash \mathscr{K}_{j}}\left[V(\lambda) e^{c(\omega)}\right]^{r} d \mu+\sigma_{j} \max _{|x| \leqslant M_{0}} g(x) .
\end{aligned}
$$

Правая часть полученного неравенства не зависит от $u \in \mathscr{U}$ и стремится к нулю при $j \rightarrow \infty$ в силу выбора $\varepsilon_{j}$ и $\sigma_{j}$ и условия н). Лемма доказана.

Следуюшая теорема характеризует степень эффективности рассматриваемой аппроксимации для задачи без фазовых ограничений.

ТЕОРема 1.3. Пусть выполнены условия леммы $1.5, \widehat{\mathscr{J}}_{j}=\inf _{u \in \mathscr{U}_{d}} \mathscr{J}_{j}(u)$ и $u_{j}$ - субоптимальнье (оптимальнье) управления для задач (1.18)-(1.21) $(j=1,2, \ldots)$ такие, что $\Delta_{j}=\mathscr{J}_{j}\left(u_{j}\right)-\widehat{\mathscr{J}}_{j} \rightarrow 0$ при $j \rightarrow \infty$. Тогда $u_{j}-\mathcal{м u - ~}$ нимизирующая последовательность управлений для функиионала качества задачи (1.1)-(1.4). 
ДокАЗАТЕльство. Заметим, прежде всего, что при отсутствии фазовых ограничений и в условиях леммы 1.5 любое удовлетворяюшее (1.3) управление допустимо как для исходной, так и для приближенных задач.

Пусть $\widehat{\mathscr{J}}=\inf _{u \in \mathcal{U}_{d}} \mathscr{J}(u), u_{\varepsilon}-$ субоптимальное (оптимальное) управление для задачи (1.1)-(1.4) такое, что $\mathscr{J}\left(u_{\varepsilon}\right)-\widehat{\mathscr{J}}<\varepsilon$. Тогда

$\mathscr{J}\left(u_{j}\right)-\widehat{\mathscr{J}}=\mathscr{J}\left(u_{j}\right)-\mathscr{J}_{j}\left(u_{j}\right)+\mathscr{J}_{j}\left(u_{j}\right)-\mathscr{J}_{j}\left(u_{\varepsilon}\right)+\mathscr{J}_{j}\left(u_{\varepsilon}\right)-\mathscr{J}\left(u_{\varepsilon}\right)+\mathscr{J}\left(u_{\varepsilon}\right)-\widehat{\mathscr{J}}$.

Поскольку $\mathscr{J}\left(u_{j}\right)-\widehat{\mathscr{J}} \geqslant 0$, нас интересует только оценка этой разности сверху. Разности $\mathscr{J}\left(u_{j}\right)-\mathscr{J}_{j}\left(u_{j}\right)$ и $\mathscr{J} j\left(u_{\varepsilon}\right)-\mathscr{J}\left(u_{\varepsilon}\right)$ стремятся к нулю при $j \rightarrow \infty$ согласно лемме 1.5. Поскольку управление $u_{\varepsilon}$ является допустимьм для задачи (1.18)-(1.21), разность $\mathscr{J}_{j}\left(u_{j}\right)-\mathscr{J}_{j}\left(u_{\varepsilon}\right) \leqslant \mathscr{J}_{j}\left(u_{j}\right)-\widehat{\mathscr{J}}_{j}=\Delta_{j} \rightarrow 0$ при $j \rightarrow \infty$ по условию теоремы. Наконец, разность $\mathscr{J}\left(u_{\varepsilon}\right)-\widehat{\mathscr{J}}$ меньше $\varepsilon$, и в силу произвольности $\varepsilon$ получаем утверждение теоремы.

Рассмотрим теперь задачу с фазовыми ограничениями (1.6). При наличии фазовых ограничений множества допустимых управлений $\mathscr{U}_{d}$ исходной и $\mathscr{U}_{d_{j}}$ приближенных задач не совпадают. Это вынуждает прибегнуть к некоторому видоизменению условий (1.23) с тем, чтобы обеспечить хотя бы включение $\mathscr{U}_{d_{j}} \subset \mathscr{U}_{d}$.

Выберем последовательность $\varepsilon_{j}$ удовлетворяющей помимо (1.26) также услоВию

$$
M_{j}^{L-1}\left(C_{j} \varepsilon_{j}+\xi_{j}\left(\varepsilon_{j}\right)\right) \rightarrow 0 \text { при } j \rightarrow \infty,
$$

где $M_{j}, C_{j}$ и $\xi_{j}$ такие же, как в $(1.25)$ и (1.26).

Пусть выполнено кроме а)-в) условие м). Для $l=1,2, \ldots, L$ и $j=1,2, \ldots$ определим константы

$$
\begin{aligned}
\nu_{l, j}= & n^{l}\left[l M_{j}^{l-1}\left(C_{j} \varepsilon_{j}+\xi_{j}\left(\varepsilon_{j}\right)\right)+R_{0}^{l} \sigma_{j}\right. \\
& \left.+\frac{\sigma_{j}^{(\rho-l) / \rho}}{D^{l / \rho_{1}}}\left(\int_{\Lambda \backslash \mathscr{K}_{j}}\left[V(\lambda) e^{c(\omega)}\right]^{\rho_{2}} d \mu\right)^{l / \rho}\right] .
\end{aligned}
$$

Из сделанных предположений следует, что $\nu_{l, j} \rightarrow 0$ при $j \rightarrow \infty, l=1,2, \ldots, L$.

Положим

$$
X_{l, j}=C\left(\left[C X_{l}\right]_{\nu_{l, j}}\right),
$$

где символами $C$ и $[\cdot]_{\nu}$ обозначаются соответственно дополнение к множеству и $\nu$-окрестность множества в метрическом пространстве.

Заменим ограничение (1.23) на

$$
\mathfrak{M}_{l, j}(\cdot) \in X_{l, j}, \quad l=1,2, \ldots, L .
$$

ТЕОРЕМА 1.4. Пусть выполнены условия леммы 1.5 и условие м), и пусть множества $X_{l}$ открыты в $\mathscr{C}_{l}$. Тогда:

1) существует $j_{0}$ такое, что при всех $j \geqslant j_{0}$ множсества $X_{l, j}$ непусть;

2) существует $j_{1} \geqslant j_{0}$ такое, что при всех $j \geqslant j_{1}$ множсество допустимых управлений задачи (1.18)-(1.21), (1.30) непусто;

3) если $u_{j}, j=j_{1}, j_{1}+1, \ldots$, - такое субоптимальное управление для задачи (1.18)-(1.21), (1.30), что $\Delta_{j}=\mathscr{J}_{j}\left(u_{j}\right)-\widehat{\mathscr{J}}_{j} \rightarrow 0$ при $j \rightarrow \infty$, то $u_{j}$ образуют минимизирующую последовательность управлений для задачи (1.1)-(1.4), (1.6). 
ДокАЗАТЕЛЬСтво. Первое утверждение теоремы является следствием открытости множеств $X_{l}$ и сходимости $\nu_{l, j}$ к нулю.

Рассмотрим, далее, произвольное управление $u \in \mathscr{U}$, и пусть $x(t)=x(t, u, \lambda)$, $\mathfrak{M}_{l}(t)=\mathrm{M}\left[\bigotimes^{l} x(t)\right]$ и $\mathfrak{M}_{l, j}(t)=\mathrm{M}_{j}\left[\bigotimes^{l} x(t)\right]$. Тогда, оценивая аналогично $(1.27)$, получаем:

$$
\begin{aligned}
\mid \mathfrak{M}_{l}(t) & -\mathfrak{M}_{l, j}(t)\left|\leqslant \int_{\mathscr{K}_{j}} \sum_{i} \gamma_{i}(\lambda)\right| \bigotimes^{l} x\left(t, \lambda_{i}\right)-\bigotimes x(t, \lambda) \mid d \mu \\
& +\int_{\Lambda \backslash \mathscr{K}_{j}}\left|\bigotimes^{l} x(t, \lambda)\right| d \mu \\
\leqslant & \ln ^{l} \int_{\mathscr{K}_{j}} \sum_{i} \gamma_{i}(\lambda)\left|x\left(t, \lambda_{i}\right)-x(t, \lambda)\right| \max _{\lambda \in \mathscr{K}_{j}}|x(t, \lambda)|^{l-1} d \mu \\
& +n^{l} \int_{\Lambda \backslash \mathscr{K}_{j}}|x(t, \lambda)|^{l} d \mu \leqslant \nu_{l, j} .
\end{aligned}
$$

Отсюда в силу определения множеств $X_{l, j}$ следует, что допустимые управления для задач (1.18)-(1.21), (1.30) будут таковыми и для задачи (1.1)-(1.4), (1.6).

С другой стороны, если $u$ - допустимое управление для исходной задачи, то в силу (1.6) и открытости множеств $X_{l}$ расстояния $\nu_{l}=\operatorname{dist}\left(\mathfrak{M}_{l}(\cdot), C X_{l}\right)$ положительны. Выбрав номер $j=j(u)$ с таким расчетом, чтобы при всех $j \geqslant j(u)$ вьполнялось $\nu_{l, j}<\nu_{l} / 2$ для $l=1,2, \ldots, L$, из (1.31) получим, что

$$
\operatorname{dist}\left(\mathfrak{M}_{l, j}, C X_{l}\right) \geqslant \operatorname{dist}\left(\mathfrak{M}_{l}, C X_{l}\right)-\left\|\mathfrak{M}_{l, j}-\mathfrak{M}_{l}\right\|_{\mathscr{C}_{l}} \geqslant \nu_{l}-\nu_{l, j}>\nu_{l, j}
$$

Отсюда в силу $(1.29) \mathfrak{M}_{l, j}(\cdot) \in X_{l, j}$ и, значит, управление $u$ допустимо для задач $(1.18)-(1.21),(1.30)$ при $j \geqslant j(u)$.

Для доказательства второго утверждения теоремы теперь остается сослаться на условие г), третье же доказывается применением выкладки, использовавшейся в теореме 1.3 .

Для задачи с фазовьм ограничением (1.5) получен следующий результат.

ТЕОРема 1.5. Предположим, что выполнены условия теоремы 1.1 и леммъ 1.5. Пусть, далее, $\widehat{\mathscr{J}}$ - инфимум функционала качества задачи (1.1)-(1.5), $\widehat{\mathscr{J}}_{j}-$ задачи (1.18)-(1.22). Тогда $\widehat{\mathscr{J}}_{j} \rightarrow \widehat{\mathscr{J}} n p u j \rightarrow \infty$.

ДокАЗАТЕЛЬство. В приводимом ниже доказательстве мы следуем схеме, предложенной А. Дончевым в $[9 ; \S 2.2]$.

Для замкнутого множества $X$ условие (1.5) эквивалентно тому, что $x(\cdot, \lambda) \in X$ $\forall \lambda \in \operatorname{supp} \mu$. Поэтому любое допустимое для задачи (1.1)-(1.5) управление будет допустимьм и для приближенных задач.

Пусть $\widehat{u}$ - оптимальное управление для исходной задачи (существующее согласно теореме 1.1). Тогда в силу леммы $1.5 \mathscr{J} j(\widehat{u}) \rightarrow \mathscr{J}(\widehat{u})=\widehat{\mathscr{J}}$ при $j \rightarrow \infty$, откуда, поскольку $\mathscr{J}_{j}(\widehat{u}) \geqslant \widehat{\mathscr{J}}_{j}$, заключаем, что

$$
\limsup _{j \rightarrow \infty} \widehat{\mathscr{J}}_{j} \leqslant \widehat{\mathscr{J}}
$$


Докажем, что

$$
\liminf _{j \rightarrow \infty} \widehat{\mathscr{J}}_{j} \geqslant \widehat{\mathscr{J}}
$$

Предположим обратное, т.е. что сушествует подпоследовательность $\left\{j^{\prime}\right\} \subset\{j\}$, для которой

$$
\lim _{j^{\prime} \rightarrow \infty} \widehat{\mathscr{J}}_{j^{\prime}}<\widehat{\mathscr{J}}
$$

Пусть $\widehat{u}_{j}$ - оптимальное управление для приближенной задачи, $\Pi_{j}-$ распределение случайного вектора $\left(\lambda, x\left(\cdot, \widehat{u}_{j}, \lambda\right)\right)$, заданного на вероятностном пространстве $\left(\Lambda, \mathscr{B}(\Lambda), \mu_{j}\right)$ :

$$
\Pi_{j}(B)=\mu_{j}\left\{\lambda:\left(\lambda, x\left(\cdot, \widehat{u}_{j}, \lambda\right)\right) \in B\right\} \quad \forall B \in \mathscr{B}(\Lambda \times \mathscr{C}) .
$$

Из леммы 1.4 следует плотность семейства мер $\mu_{j}$, откуда так же, как в лемме 1.2 , вьводится плотность и, значит, слабая компактность семейства $\Pi_{j}$.

Повторяя рассуждения теоремы 1.1 , устанавливаем сушествование меры П и удовлетворяющего (1.3) управления $\bar{u}$ таких, что $\Pi_{j} \rightarrow$ П слабо на $\Lambda \times \mathscr{C}$ и $\chi_{i}\left(\cdot, \widehat{u}_{j}(\cdot)\right) \rightarrow \chi_{i}(\cdot, \bar{u}(\cdot)), i=1, \ldots, m$, слабо $(*$-слабо $)$ в $L_{p_{i}}(0,1)$ по некоторой подпоследовательности $j=j^{\prime \prime} \rightarrow \infty,\left\{j^{\prime \prime}\right\} \subset\left\{j^{\prime}\right\}$.

Пусть $\mathscr{A}_{u}$ - оператор (1.10), $\pi$ - проекция $\Lambda \times \mathscr{C} \rightarrow \Lambda$. Согласно лемме 1.3 $\mathscr{A}_{\widehat{u}_{j}}^{*} \Pi_{j}=\delta_{0}$ и $\pi^{*} \Pi_{j}=\mu_{j}$. Переходя в обоих равенствах к пределу при $j=j^{\prime \prime} \rightarrow \infty$, с учетом леммы 1.4 получаем $\mathscr{A} \frac{*}{u} \Pi=\delta_{0}$ и $\pi^{*} \Pi=\mu$. Последнее означает (лемма 1.3) совпадение П с распределением П $\bar{u}$, задаваемым формулой (1.9). Поэтому аналогично (1.13)

$$
\mathscr{J}(\bar{u})=\int g(x(1)) d \Pi \leqslant \liminf _{j^{\prime \prime} \rightarrow \infty} \int g(x(1)) d \Pi_{j^{\prime \prime}}=\lim _{j^{\prime} \rightarrow \infty} \widehat{\mathscr{J}}_{j^{\prime}} .
$$

Покажем теперь, что для $x(t, \bar{u})$ вьполняется фазовое ограничение $(1.5)$ и, значит, $\bar{u} \in \mathscr{U}_{d}$. Для этого достаточно проверить выполнение условия (1.14):

$$
\Pi_{\bar{u}}(\Lambda \times X) \geqslant \limsup _{j^{\prime \prime} \rightarrow \infty} \Pi_{j^{\prime \prime}}(\Lambda \times X)=\limsup _{j^{\prime \prime} \rightarrow \infty} \mu_{j^{\prime \prime}}(\Lambda)=1 .
$$

Таким образом, $\widehat{\mathscr{J}} \leqslant \mathscr{J}(\bar{u}) \leqslant \lim _{j^{\prime} \rightarrow \infty} \widehat{\mathscr{J}}_{j^{\prime}}$ в силу (1.35), что противоречит предположению (1.34) и тем самьм доказьвает (1.33). Наконец, из (1.32) и (1.33) следует утверждение теоремы.

Аналогичный доказанному результат справедлив для задачи с фазовым ограничением (1.6) в случае замкнутых множеств $X_{l}$. Для обеспечения включения $\mathscr{U}_{d} \subset \mathscr{U}_{d_{j}} \forall j$, сушественно использовавшегося в доказательстве, заменим фазовые ограничения (1.23) в приближенных задачах на

$$
\mathfrak{M}_{l, j}(\cdot) \in \overline{\left[X_{l}\right]_{\nu_{l, j}}}
$$

где $\nu_{l, j}$ - константы из (1.28), черта над знаком множества означает его замыкание. Потребуем дополнительно непрерывности функции Ляпунова $V(x, \omega)$ по совокупности аргументов и при построении приближенных мер $\mu_{j}$ последовательность $\varepsilon_{j}$ выберем удовлетворяющей помимо (1.26) также условию

$$
m_{V}^{j}\left(\left(C_{j}+1\right) \varepsilon_{j}+\xi_{j}\left(\varepsilon_{j}\right)\right) \leqslant c_{0}=\mathrm{const} \quad \forall j,
$$

где $m_{V}^{j}(\varepsilon)=\max \left\{\left|V(\lambda)-V\left(\lambda^{\prime}\right)\right|: \lambda, \lambda^{\prime} \in B_{M_{j}} \times K_{j}, \rho_{\Lambda}\left(\lambda, \lambda^{\prime}\right) \leqslant \varepsilon\right\}-$ модуль непрерывности $V(\lambda)$ на компакте $B_{M_{j}} \times K_{j}$, причем $C_{j}, \xi_{j}, K_{j}$ и $M_{j}$ здесь те же, что и в $(1.25),(1.26)$. Разумеется, вьполнения (1.37) можно добиться при любой константе $c_{0}>0$, поскольку $m_{V}^{j}(\varepsilon) \rightarrow 0$ при $\varepsilon \rightarrow 0$. 
ТЕОРема 1.6. Пусть выполнены условия теоремы 1.2 и леммы 1.5, а такжсе условие (1.37). Пусть, далее, $\widehat{\mathscr{J}}$ - инфимум функчионала качества задачи (1.1)-(1.4), (1.6), $\widehat{\mathscr{J}}_{j}$ - задачи (1.18)-(1.21), (1.36). Тогда $\widehat{\mathscr{J}}_{j} \rightarrow \widehat{\mathscr{J}} n p u$ $j \rightarrow \infty$.

Доказательство состоит из соответствующим образом модифицированных фрагментов доказательств теорем 1.5 и 1.2 и потому опускается.

В заключение приведем выражения для констант $M$ и $C$ и функции $\xi(\varepsilon)$, введенных в лемме 1.1 и используемых при аппроксимации:

$$
M=\sup \{|x|: \exists \omega \in K \quad \text { такое, что } V(x, \omega) \leqslant T\},
$$

где $T=\sup \left\{V\left(x_{0}, \omega\right) e^{c(\omega)}: x_{0} \in B_{R}, \omega \in K\right\}$

$$
C=\exp \left\{\int_{0}^{1} d_{M, K}(\tau) d \tau\right\}
$$

где $d_{M, K}(\tau)$ - функция из условия б); наконец,

$$
\xi(\varepsilon)=C \int_{0}^{1} m_{\varphi, M, K}(\tau, \varepsilon) d \tau
$$

где

$$
\begin{aligned}
m_{\varphi, M, K}(\tau, \varepsilon)= & \sup \left\{\left|\varphi(\tau, x, u, \omega)-\varphi\left(\tau, x, u, \omega^{\prime}\right)\right|:\right. \\
& \left.u \in U, x \in B_{M}, \omega, \omega^{\prime} \in K, \rho_{\Omega}\left(\omega, \omega^{\prime}\right) \leqslant \varepsilon\right\} .
\end{aligned}
$$

\section{§2. Задачи с распределенными параметрами}

В настоящем разделе показывается, что методы $\S 1$ могут быть применены в ситуации, когда динамическая система описывается уравнением с частными производными, имеющим случайные параметры. Не ставя себе цели получения сколько-нибудь общих результатов, проведем все исследование на примере одного нелинейного гиперболического уравнения, изучавшегося в [10] и ряде последующих работ.

Пусть $x=\left(x_{1}, \ldots, x_{n}\right) \in \mathbb{R}^{n}, t \in \mathbb{R}, \mathscr{O} \subset \mathbb{R}^{n}$ - ограниченная область с границей $\partial \mathscr{O}$ класса $C^{\infty}, Q$ - цилиндр $(0,1) \times \mathscr{O} \subset \mathbb{R}_{t} \times \mathbb{R}_{x}^{n}, \Delta=\frac{\partial^{2}}{\partial x_{1}^{2}}+\cdots+\frac{\partial^{2}}{\partial x_{n}^{2}}-$ оператор Лапласа, $\rho>0$ - константа. Рассматривается краевая задача

$$
\begin{gathered}
y_{t t}^{\prime \prime}(t, x)-\Delta y+|y|^{\rho} y=F(t, x), \quad(t, x) \in Q, \\
\left.y\right|_{t=0}=y_{0}(x),\left.\quad y_{t}^{\prime}\right|_{t=0}=y_{1}(x), \\
\left.y\right|_{x \in \partial \mathscr{O}}=0,
\end{gathered}
$$

где $F, y_{0}$ и $y_{1}-$ заданные функции.

Пусть $H^{s}=H^{s}(\mathscr{O})$ и $\stackrel{\circ}{H}^{s}=\stackrel{\circ}{H}{ }^{s}(\mathscr{O})$ - пространства Соболева [8] с нормой $\|\cdot\|_{s}$, $L_{p}=L_{p}(\mathscr{O})$ - лебегово пространство, норма в котором будет обозначаться символом $|\cdot|_{p}$. Для нормы и скалярного произведения в пространстве $L_{2}$ используются соответственно обозначения $|\cdot|$ и $(\cdot, \cdot)$. 
Обозначим $V=\stackrel{\circ}{H}{ }^{1} \cap L_{\rho+2}, H=L_{2}, V^{\prime}=H^{-1}+L_{(\rho+2) /(\rho+1)}$ - пространство, сопряженное к $V$. Введем пространство траекторий задачи (2.1)-(2.3)

$$
Y=\left\{\varphi=\varphi(t) \in L_{\infty}(0,1 ; V): \varphi^{\prime} \in L_{\infty}(0,1 ; H), \varphi^{\prime \prime} \in L_{2}\left(0,1 ; V^{\prime}\right)\right\}
$$

и функционал энергии

$$
E(\varphi, \psi)=\frac{1}{2}\|\varphi\|_{1}^{2}+\frac{1}{\rho+2}|\varphi|_{\rho+2}^{\rho+2}+\frac{1}{2}|\psi|^{2},
$$

где $\varphi \in V, \psi \in H$.

Мы будем опираться на полученные в [10] результаты о существовании, единственности и непрерывной зависимости от исходных данных решений задачи (2.1)-(2.3), сведя их для компактности в одну теорему.

Теорема 2.1 (Лионс [10]). Пусть $y_{0} \in V, y_{1} \in H, F \in L_{2}(Q)$. Тогда задача (2.1)-(2.3) обладает решением $y$, принадлежсащим пространству $Y$ и удовлетворяющим әнергетическому неравенству

$$
\begin{gathered}
E\left(y(t), y_{t}^{\prime}(t)\right) \leqslant E\left(y_{0}, y_{1}\right)+\int_{0}^{t}\left(F(\tau), y_{t}^{\prime}(\tau)\right) d \tau \\
\forall t \in[0,1]
\end{gathered}
$$

Если $\rho$ удовлетворяет условию:

$$
\begin{array}{lll}
\rho \leqslant 2 /(n-2) & n p u & n \geqslant 3, \\
\rho \quad \text { произвольно } & \text { nри } & n=1,2,
\end{array}
$$

то решение задачи (2.1)-(2.3) единственно в классе $Y$, а отобрахсение

$$
\left(y_{0}, y_{1}, F\right) \rightarrow y\left(y_{0}, y_{1}, F\right)
$$

сопоставляющее начальным условиям и правой части задачи (2.1)-(2.3) ее решение, действует непрерывно из $V \times H \times L_{2}(Q)$ в $Y$. При этом для произвольных $y_{0}, \widetilde{y}_{0} \in V, y_{1}, \widetilde{y}_{1} \in H$ u $F, \widetilde{F} \in L_{2}(Q)$ решения $y=y\left(y_{0}, y_{1}, F\right) u$ $\widetilde{y}=y\left(\widetilde{y}_{0}, \widetilde{y}_{1}, \widetilde{F}\right)$ удовлетворяют неравенству

$$
\begin{aligned}
\| y(t) & -\widetilde{y}(t) \|_{1}^{2}+\left|y_{t}^{\prime}(t)-\widetilde{y}_{t}^{\prime}(t)\right|^{2} \\
& \leqslant C(y, \widetilde{y})\left(\left\|y_{0}-\widetilde{y}_{0}\right\|_{1}^{2}+\left|y_{1}-\widetilde{y}_{1}\right|^{2}+\|F-\widetilde{F}\|_{L_{2}(Q)}^{2}\right) \quad \forall t \in[0,1],
\end{aligned}
$$

әде функиия $C(y, \widetilde{y})$ ограничена на ограниченных подмножсествах $Y$.

ЗАмечАниЕ 2.1. В силу теоремы вложения Соболева при выполнении условия (2.6) $\stackrel{\circ}{H}{ }^{1} \subset L_{\rho+2}$ и потому $V=\stackrel{\circ}{H^{1}}$ и, соответственно, $V^{\prime}=H^{-1}$.

В дальнейшем условие (2.6) предполагается выполненным.

Нам понадобится следуюшая

ЛЕмма 2.1. Пространство $Y$ вполне непрерьвно вложено в пространство $Z=\left\{\varphi \in C\left(0,1 ; L_{q}\right): \varphi^{\prime} \in C\left(0,1 ; V^{\prime}\right)\right\}$, где $q=\max \{2, \rho+1\}$. 
ДокАЗАТЕЛЬство. Достаточно установить компактность вложений

$$
\begin{gathered}
Y \subset C\left(0,1 ; L_{q}\right), \\
\left\{\psi \in L_{\infty}(0,1 ; H): \psi^{\prime} \in L_{2}\left(0,1 ; V^{\prime}\right)\right\} \subset C\left(0,1 ; V^{\prime}\right) .
\end{gathered}
$$

Компактность второго вложения непосредственно вытекает из теоремы Дубинского [5; гл. IV, теорема 4.1]. Докажем полную непрерьвность первого вложения.

Согласно теореме вложения Соболева при выполнении (2.6) $V$ вполне непрерывно вложено в $L_{q}$ (в [11] показано, что это верно и в отсутствие условия (2.6)). Поэтому в силу интерполяционного неравенства [8; гл. 1, теорема 16.4] для произвольного $\eta>0$ найдется константа $C_{\eta}$ такая, что при всех $y \in Y, t, t^{\prime} \in[0,1]$

$$
\begin{aligned}
\left|y(t)-y\left(t^{\prime}\right)\right|_{q} & \leqslant \eta\left\|y(t)-y\left(t^{\prime}\right)\right\|_{V}+C_{\eta}\left|y(t)-y\left(t^{\prime}\right)\right| \\
& \leqslant 2 \eta\|y\|_{L_{\infty}(0,1 ; V)}+C_{\eta}\left|t-t^{\prime}\right|\left\|y_{t}^{\prime}\right\|_{L_{\infty}(0,1 ; H)} .
\end{aligned}
$$

Таким образом, любое ограниченное в $Y$ семейство функций равностепенно непрерывно в $C\left(0,1 ; L_{q}\right)$, и компактность вложения $Y \subset C\left(0,1 ; L_{q}\right)$ следует из теоремы Дубинского.

Ниже пространство $Y$ будет снабжаться двумя топологиями: естественной, порожденной нормой

$$
\|\varphi\|_{Y}=\|\varphi\|_{L_{\infty}(0,1 ; V)}+\left\|\varphi^{\prime}\right\|_{L_{\infty}(0,1 ; H)}+\left\|\varphi^{\prime \prime}\right\|_{L_{2}\left(0,1 ; V^{\prime}\right)},
$$

и индуцированной вложением $Y \subset Z$, т.е. порожденной нормой

$$
\|\varphi\|_{Z}=\|\varphi\|_{C\left(0,1 ; L_{q}\right)}+\left\|\varphi^{\prime}\right\|_{C\left(0,1 ; V^{\prime}\right)} .
$$

Пространство $Y$, снабженное нормой (2.8), будем обозначать через $\widetilde{Y}$, оставив обозначение $Y$ для естественной топологии. Очевидно, $\widetilde{Y}$ - сепарабельное метрическое (неполное) пространство.

Поставим задачу оптимального управления решением динамической системы (2.1)-(2.3), предполагая, что управляющая функция $u(t, x)$ аддитивньм образом входит в правую часть $F(t, x)$ уравнения $(2.1)$, т.е.

$$
F(t, x)=u(t, x)+f(t, x),
$$

и удовлетворяет ограничению

$$
u \in \mathscr{U},
$$

где $\mathscr{U}$ - некоторое ограниченное подмножество $L_{2}(Q)$. Функции $f \in L_{2}(Q), y_{0} \in V$ и $y_{1} \in H$ в дальнейшем предполагаются случайньми, а функционал качества задается в виде

$$
\mathscr{J}(u)=\mathrm{M}\left\|y(1)-z_{0}\right\|_{1}^{2} \rightarrow \inf ,
$$

где $z_{0}$ - некоторая фиксированная функция из $\stackrel{\circ}{H^{1}}, \mathrm{M}$ - оператор математического ожидания.

Пусть $\Lambda=V \times H \times L_{2}(Q)$ - пространство случайных параметров задачи (2.1)-(2.3), $\mu$ - распределение на $\mathscr{B}(\Lambda)$ случайного вектора $\lambda=\left(y_{0}, y_{1}, f\right)$. Для произвольного управления $u \in L_{2}(Q)$ и вектора $\lambda \in \Lambda$ обозначим через $y(u, \lambda)$ отвечаюшее им решение задачи (2.1)-(2.3). Пусть, наконец, $\Pi_{u}$ - распределение случайного вектора $(\lambda, y(u, \lambda))$ :

$$
\Pi_{u}(B)=\mu\{\lambda:(\lambda, y(u, \lambda)) \in B\}, \quad B \in \mathscr{B}(\Lambda \times \tilde{Y}) .
$$


Лемма 2.2. Семейство мер $\left\{\Pi_{u}, u \in \mathscr{U}\right\}$ слабо компактно на $\Lambda \times \tilde{Y}$.

ДокАЗАТЕльство. Заметим, прежде всего, что из неравенства (2.5) в силу леммы Гронуолла следует оценка

$$
\begin{aligned}
E\left(y(t), y_{t}^{\prime}(t)\right) & \leqslant e^{t}\left(E\left(y_{0}, y_{1}\right)+\frac{1}{2}\|F\|_{L_{2}(Q)}^{2}\right) \\
& \leqslant e\left(E\left(y_{0}, y_{1}\right)+\|u\|_{L_{2}(Q)}^{2}+\|f\|_{L_{2}(Q)}^{2}\right), \quad t \in[0,1] .
\end{aligned}
$$

Согласно (2.1) $y_{t t}^{\prime \prime}=\Delta y-|y|^{\rho} y+F$. Отсюда получим, что при п.в. $t \in(0,1)$

$$
\left\|y_{t t}^{\prime \prime}(t)\right\|_{V^{\prime}}=\sup _{\left\{v \in V,\|v\|_{V}=1\right\}}\left|\left(y_{t t}^{\prime \prime}(t), v\right)\right| \leqslant\|y(t)\|_{1}+|y(t)|_{\rho+2}^{\rho+1}+|F(t)|
$$

и потому с учетом (2.4)

$$
\left\|y_{t t}^{\prime \prime}\right\|_{L_{2}\left(0,1 ; V^{\prime}\right)}^{2} \leqslant C_{\rho}\left[1+\left(\sup _{t} E\left(y(t), y_{t}^{\prime}(t)\right)\right)^{1+\rho /(\rho+2)}+\|u\|_{L_{2}(Q)}^{2}+\|f\|_{L_{2}(Q)}^{2}\right]
$$

где константа $C_{\rho}$ не зависит от $y$.

Для произвольного $\varepsilon>0$ возьмем компакт $K_{\varepsilon} \subset \Lambda$ такой, что $\mu\left(K_{\varepsilon}\right)>1-\varepsilon$. Тогда в силу (2.13) и (2.14) сушествует такое $R=R_{\varepsilon}>0$, что для любых $\lambda \in K_{\varepsilon}$ и $u \in \mathscr{U} y(u, \lambda) \in B_{R}=\left\{\|y\|_{Y} \leqslant R\right\}$ (напомним, что $\mathscr{U}$ ограничено в $\left.L_{2}(Q)\right)$.

Согласно лемме 2.1 шар $B_{R}$ предкомпактен в $Z$. Поскольку $B_{R}$ компактен в *-слабой топологии $Y$, то из любой сходящейся в $Z$ последовательности $\left\{z_{n}\right\} \subset B_{R}$ можно выбрать $*$-слабо сходяшуюся в $Y$ (к тому же пределу) подпоследовательность. Значит, $B_{R}$ замкнут и, следовательно, компактен в $Z$. Учитывая, что $B_{R} \subset \widetilde{Y}$ и пространство $\widetilde{Y}$ снабжено топологией $Z$, заключаем, что $B_{R}$ компактен также и в $\widetilde{Y}$.

Заметим теперь, что в силу (2.12) для произвольного $u \in \mathscr{U} \Pi_{u}\left(K_{\varepsilon} \times B_{R}\right)=$ $\mu\left\{\lambda \in K_{\varepsilon}: y(u, \lambda) \in B_{R}\right\}=\mu\left(K_{\varepsilon}\right)>1-\varepsilon$, и потому утверждение леммы следует из теоремы Прохорова.

Для произвольного $u \in L_{2}(Q)$ введем оператор

$$
\begin{gathered}
\mathscr{A}_{u}(\lambda, y)=\left(y_{t t}^{\prime \prime}-\Delta y+|y|^{\rho} y-u-f, y(0)-y_{0}, y_{t}^{\prime}(0)-y_{1}\right), \\
\lambda=\left(y_{0}, y_{1}, f\right) \in \Lambda, \quad y \in Y .
\end{gathered}
$$

Оператор $\mathscr{A}_{u}$ действует непрерьвно из $\Lambda \times \widetilde{Y}$ в пространство $H^{-s}(Q) \times L_{q} \times V^{\prime}$, где $s=[(n+3) / 2]$ - целая часть числа $(n+3) / 2$ (при рассмотрении слагаемого $|y|^{\rho} y$ ссылаемся на теорему вложения Соболева).

Пусть $\pi$ - проекция $(\lambda, y) \rightarrow \lambda, \Lambda \times \widetilde{Y} \rightarrow \Lambda$. Справедлив аналог леммы 1.3 , получаемый дословньм повторением ее доказательства:

ЛЕмма 2.3. Борелевская мера П на $\Lambda \times \widetilde{Y}$ совпадает с распределением $\Pi_{u}$, $u \in L_{2}(Q)$, тогда и только тогда, когда

$$
\mathscr{A}_{u}^{*} \Pi=\delta_{0} \quad u \quad \pi^{*} \Pi=\mu,
$$


где $\delta_{0}-$ единичная мера, сосредоточенная в нуле пространства $H^{-s}(Q) \times$ $L_{q} \times V^{\prime}$.

Назовем управление допустимым, если $u \in \mathscr{U}$ и $\mathscr{J}(u)<\infty$. (Пример вьполнения второго условия: пусть

$$
\mathrm{M}\left[E\left(y_{0}, y_{1}\right)+\|f\|_{L_{2}(Q)}^{2}\right]<\infty .
$$

Тогда в силу оценки $(2.13) \mathscr{J}(u)<\infty \forall u \in L_{2}(Q)$.)

Будем предполагать, что множество допустимых управлений непусто.

По той же схеме, что и теоремы существования в $\S 1$, доказьвается

ТЕОРема 2.2. Пусть мнохсество $\mathscr{U}$ выпукло и замкнуто в $L_{2}(Q)$. Тогда существует по меньшей мере одно оптимальное управление для задачи (2.1)-(2.3), (2.9)-(2.11)

ДокАЗАТЕЛЬСтво. Возьмем минимизирующую последовательность управлений $u_{n} \in \mathscr{U}$, и пусть $\Pi_{n}=\Pi_{u_{n}}$ - распределения (2.12). Перейдя к подпоследовательности, можем считать, что меры $\Pi_{n}$ слабо на $\Lambda \times \widetilde{Y}$ сходятся к некоторой мере П, а управления $u_{n}$ слабо в $L_{2}(Q)$ (и, следовательно, сильно в $\left.H^{-s}(Q)\right)$ сходятся к некоторой функции $\widehat{u}$. Заметим сразу, что благодаря выпуклости множество $\mathscr{U}$ слабо замкнуто в $L_{2}(Q)$, а потому $\widehat{u} \in \mathscr{U}$.

Так же как в теореме 1.1, устанавливается, что $\Pi=\Pi_{\widehat{u}}$. Действительно, из сходимости $\left(\lambda_{k}, y_{k}\right) \rightarrow(\lambda, y)$ в $\Lambda \times \widetilde{Y}$ и $u_{k} \rightarrow u$ в $H^{-s}(Q)$ следует, что $\mathscr{A}_{u_{k}}\left(\lambda_{k}, y_{k}\right) \rightarrow$ $\mathscr{A}_{u}(\lambda, y)$ в $H^{-s}(Q) \times L_{q} \times V^{\prime}$ - это вытекает непосредственно из структуры $(2.15)$ оператора $\mathscr{A}_{u}$. Поэтому, переходя к пределу в соотношениях $\mathscr{A}_{u_{n}}^{*} \Pi_{n}=\delta_{0}$ и $\pi^{*} \Pi_{n}=\mu$, справедливых в силу леммы 2.3, получаем $\mathscr{A}_{\widehat{u}}^{*} \Pi=\delta_{0}$ и $\pi^{*} \Pi=\mu$ и, значит, $\Pi=\Pi_{\widehat{u}}$.

Покажем, что $\widehat{u}$ - оптимальное управление. Для этого заметим, что функционал $\Phi(y)=\left\|y(1)-z_{0}\right\|_{1}^{2}$ полунепрерывен снизу на $\widetilde{Y}$. Действительно, пусть $y_{k} \in A_{c}=$ $\{y \in \widetilde{Y}: \Phi(y) \leqslant c\}$ и $y_{k} \rightarrow y$ в $\widetilde{Y}$. Тогда в силу компактности шара $\left\{\left\|z-z_{0}\right\|_{1}^{2} \leqslant c\right\}$ в слабой топологии пространства $H^{1}$ найдутся подпоследовательность $\left\{k^{\prime}\right\} \subset\{k\}$ и функция $v \in H^{1}$ такие, что $y_{k^{\prime}}(1) \rightarrow v$ слабо в $H^{1}$ и $\left\|v-z_{0}\right\|_{1}^{2} \leqslant c$. Учитывая сходимость $y_{k}(1) \rightarrow y(1)$ в $L_{q}$, заключаем, что $v=y(1)$. Следовательно, $y \in A_{c}$ и потому $A_{c}$ замкнуто в $\widetilde{Y}$.

Из полунепрерывности снизу функционала $\Phi(y)$ следует, что

$$
\mathscr{J}(\widehat{u})=\int \Phi(y) d \Pi \leqslant \liminf _{n \rightarrow \infty} \int \Phi(y) d \Pi_{n}=\liminf _{n \rightarrow \infty} \mathscr{J}\left(u_{n}\right)=\inf \mathscr{J}(u),
$$

и потому $\widehat{u}$ - оптимальное управление.

Аналогично $\S 1$ могут быть получены результаты об аппроксимации.

Для каждого $j \in \mathbb{N}$ выберем в $\Lambda$ такой компакт $\mathscr{K}_{j}$, что $\mu\left(\mathscr{K}_{j}\right)>1-1 / j$. Положим

$$
C_{j}=\sup _{\substack{\lambda, \widetilde{\lambda} \in \mathscr{K}_{j} \\ u \in \mathscr{U}}}\left[(C(y(u, \lambda), y(u, \widetilde{\lambda})))^{1 / 2} \cdot\left\|y(1, u, \lambda)-z_{0}\right\|_{1}\right],
$$

где $y(u, \lambda)=y(t, u, \lambda)$ - соответствуюшее управлению $u$ и вектору исходных данных $\lambda$ решение задачи $(2.1)-(2.3), C(y, \widetilde{y})$ - константа из оценки (2.7). В силу теоремы $2.1 C_{j}<\infty$. 
В каждом из компактов $\mathscr{K}_{j}$ возьмем $\frac{\varepsilon_{j}}{2}$-сеть $\left\{\lambda_{1}, \lambda_{2}, \ldots, \lambda_{N_{j}}\right\}$, причем $\varepsilon_{j}$ выберем с таким расчетом, чтобы

$$
C_{j} \varepsilon_{j} \rightarrow 0 \text { при } j \rightarrow \infty .
$$

Так же как в $\S 1$, построим меры

$$
\mu_{j}=\sum_{i=1}^{N_{j}} \alpha_{i} \delta_{\lambda_{i}},
$$

где $\alpha_{i}$ определены в (1.17). Согласно лемме $1.4 \mu_{j} \rightarrow \mu$ слабо на $\Lambda$.

Рассмотрим последовательность приближенных задач

$$
\begin{gathered}
\left(y_{i}\right)_{t t}^{\prime \prime}-\Delta y_{i}+\left|y_{i}\right|^{\rho} y_{i}=u+f_{i}, \quad i=1, \ldots, N_{j}, \\
y_{i}(0)=y_{0_{i}}, \quad\left(y_{i}\right)_{t}^{\prime}(0)=y_{1_{i}}, \\
\left.y_{i}\right|_{x \in \partial \mathscr{O}}=0, \\
u \in \mathscr{U}, \\
\mathscr{J}_{j}(u)=\sum_{i=1}^{N_{j}} \alpha_{i}\left\|y_{i}(1)-z_{0}\right\|_{1}^{2} \rightarrow \inf ,
\end{gathered}
$$

где $y_{i}=y\left(u, \lambda_{i}\right), \lambda_{i}=\left(y_{0_{i}}, y_{1_{i}}, f_{i}\right), i=1, \ldots, N_{j}$.

Справедлива

Лемма 2.4. Пусть выполнено условие (2.16). Тогда равномерно по и $\in \mathscr{U}$ $\mathscr{J}_{j}(u) \rightarrow \mathscr{J}(u)$ при $j \rightarrow \infty$.

ДокаЗАТЕЛЬСтво. Оценим разность

$$
\begin{aligned}
& \mathscr{J}_{j}(u)-\mathscr{J}(u)=\int_{\mathscr{K}_{j}}\left\|y(1)-z_{0}\right\|_{1}^{2}\left(d \mu_{j}-d \mu\right)-\int_{\Lambda \backslash \mathscr{K}_{j}}\left\|y(1)-z_{0}\right\|_{1}^{2} d \mu \\
& =\sum_{i=1}^{N_{j}} \int_{\mathscr{K}_{j}} \gamma_{i}(\lambda)\left(\left\|y_{i}(1)-z_{0}\right\|_{1}^{2}-\left\|y(1)-z_{0}\right\|_{1}^{2}\right) d \mu-\int_{\Lambda \backslash \mathscr{K}_{j}}\left\|y(1)-z_{0}\right\|_{1}^{2} d \mu,
\end{aligned}
$$

где обозначено $y(t)=y(t, u, \lambda), y_{i}(t)=y\left(t, u, \lambda_{i}\right)$.

Поскольку

$$
\left|\left\|y_{i}(1)-z_{0}\right\|_{1}^{2}-\left\|y(1)-z_{0}\right\|_{1}^{2}\right| \leqslant\left\|y_{i}(1)-y(1)\right\|_{1}\left(\left\|y_{i}(1)-z_{0}\right\|_{1}+\left\|y(1)-z_{0}\right\|_{1}\right),
$$

из $(2.7),(2.17)$ и $(2.13)$ получим

$$
\begin{aligned}
\mid \mathscr{J}_{j}(u) & -\mathscr{J}(u) \mid \leqslant \sum_{i=1}^{N_{j}} \int_{\mathscr{K}_{j}} \gamma_{i}(\lambda)\left\|\lambda-\lambda_{i}\right\|_{\Lambda}\left(C\left(y, y_{i}\right)\right)^{1 / 2}\left(\left\|y(1)-z_{0}\right\|_{1}\right. \\
& \left.+\left\|y_{i}(1)-z_{0}\right\|_{1}\right) d \mu+2 \int_{\Lambda \backslash \mathscr{K}_{j}}\left(E\left(y(1), y_{t}^{\prime}(1)\right)+\left\|z_{0}\right\|_{1}^{2}\right) d \mu \\
\leqslant & 2 C_{j} \varepsilon_{j} \mu\left(\mathscr{K}_{j}\right)+2 e \int_{\Lambda \backslash \mathscr{K}_{j}}\left(E\left(y_{0}, y_{1}\right)+\|f\|_{L_{2}(Q)}^{2}+\|u\|_{L_{2}(Q)}^{2}+\left\|z_{0}\right\|_{1}^{2}\right) d \mu \\
\leqslant & 2 C_{j} \varepsilon_{j}+2 e \int_{\Lambda \backslash \mathscr{K}_{j}}\left(E\left(y_{0}, y_{1}\right)+\|f\|_{L_{2}(Q)}^{2}\right) d \mu+\frac{2 e}{j}\left(\sup _{\mathcal{U}}\|u\|_{L_{2}(Q)}^{2}+\left\|z_{0}\right\|_{1}^{2}\right) .
\end{aligned}
$$

Учитывая (2.18), (2.16) и ограниченность $\mathscr{U}$, выводим отсюда утверждение леммы.

Обозначим $\widehat{\mathscr{J}}_{j}=\inf \mathscr{U} \mathscr{J}_{j}(u)$. Справедлива 
ТЕОРема 2.3. Предположсим, что выполнено условие (2.16). Пусть $u_{j}$, $j=1,2, \ldots$, - субоптимальные (оптимальные) управления для приближсенных задач (2.19)-(2.23) такие, что $\Delta_{j}=\mathscr{J}_{j}\left(u_{j}\right)-\widehat{\mathscr{J}}_{j} \rightarrow 0$ при $j \rightarrow \infty$. Тог-

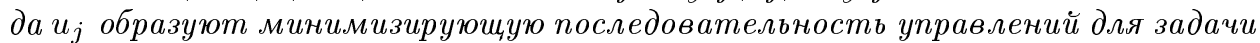
$(2.1)-(2.3),(2.9)-(2.11)$.

Доказательство опирается на лемму 2.4 и повторяет доказательство теоремы 1.3.

ЗАмечАниЕ 2.2. Задача (2.1)-(2.3), (2.9)-(2.11) может быть дополнена фазовыми ограничениями вида (1.5) или (1.6); для получаемых задач справедливы аналоги соответствующих теорем из $\S 1$.

\section{Список литературы}

1. Филиппов A. Ф. О некоторых вопросах теории оптималього регулирования // Вестн. МГУ. Сер. 1. Матем., мех., астрон. 1959. № 2. С. 25-32.

2. Экланд И., Темам Р. Выпуклый анализ и вариационные проблемы. М.: Мир, 1979.

3. Докучаев Н. Г., Якубович В. А. Принцип максимума для стохастических дифференциальных уравнений с детерминированньм управлением // Кибернетика и вычислительная техника. № 54. Киев: Наукова думка, 1982. С. 72-78.

4. Лионс Ж.-Л. Оптимальное управление системами, описьваемыми уравнениями с частными производньпи. М.: Мир, 1972.

5. Вишик М.И., Фурсиков А.В. Математические задачи статистической гидромеханики. М.: Наука, 1980

6. Хрычёв Д. А. Об одном стохастическом квазилинейном гиперболическом уравнении // Матем. сб. 1981. Т. 116. №3. С. 398-426.

7. Биллингсли П. Сходимость вероятностных мер. М.: Наука, 1977.

8. Лионс Ж.-Л., Мадженес Э. Неоднородные граничные задачи и их приложения. М.: Мир, 1971.

9. Дончев А. Системы оптимального управления. М.: Мир, 1987.

10. Лионс Ж.-Л. Некоторые методы решения нелинейных краевых задач. М.: Мир, 1972.

11. Вишик М.И., Комеч А.И. О разрешимости задачи Коши для уравнения Хопфа, соответствуюшего нелинейному гиперболическому уравнению // Труды сем. им. И.Г. Петровского. №3. М.: Изд-во МГУ, 1981. С. 19-42.

Московский институт радиотехники, электроники и автоматики (технический университет)
Поступила в редакцию 29.02 .2000 